\title{
Mutations in GFPT1-related congenital myasthenic syndromes are associated with synaptic morphological defects and underlie a tubular aggregates myopathy with synaptopathy
}

Stéphanie Bauché ${ }^{1 *}$, Geoffroy Vellieux ${ }^{1}$, Damien Sternberg ${ }^{1,2}$, Marie-Joséphine Fontenille ${ }^{1}$, Elodie De Bruyckere ${ }^{1}$, Claire-Sophie Davoine ${ }^{1}$, Guy Brochier ${ }^{3,4}$, Julien Messéant ${ }^{1}$, Lucie Wolf ${ }^{6}$, Michel Fardeau ${ }^{3,4}$, Emmanuelle Lacène ${ }^{3,4}$, Norma Romero ${ }^{3,4}$, Jeanine Koenig ${ }^{1}$, Emmanuel Fournier ${ }^{1,3,7}$, Daniel Hantai ${ }^{1}$, Nathalie Streichenberger ${ }^{8}$, Veronique Manel ${ }^{9}$, Arnaud Lacour ${ }^{10}$, Aleksandra Nadaj-Pakleza ${ }^{11}$, Sylvie Sukno ${ }^{12}$,

Françoise Bouhour ${ }^{13}$, Pascal Laforêt ${ }^{3,4,5}$, Bertrand Fontaine ${ }^{1,3}$, Laure Strochlic ${ }^{1}$, Bruno Eymard ${ }^{1,3,4}$, Frédéric Chevessier $^{6}$, Tanya Stojkovic ${ }^{3,4,5^{*}} \&$ Sophie Nicole ${ }^{1}$

1 Inserm U 1127, CNRS UMR 7225, Sorbonne Universités, UPMC Université Paris 06 UMR S 1127, Institut du Cerveau et de la Moelle épinière, ICM, 75013 Paris, France

2 APHP, UF Cardiogénétique et Myogénétique, Service de Biochimie Métabolique, Groupe Hospitalier PitiéSalpêtrière, Paris, France

3 AP-HP, Hôpital Pitié-Salpêtrière, 75013 Paris, France

4 Unité de pathologies neuromusculaires, Institut de Myologie, Sorbonne Universités, UPMC Université Paris 06 UMRS 974, Inserm U974, CNRS UMR 7215, 75013 Paris, France

5 Sorbonne Universités, UPMC Univ Paris 06, INSERM UMRS974, CNRS FRE3617, Center of Research in Myology, Myology Institute F-75013 Paris, France.

6 Institute of Neuropathology, University-Hospital Erlangen, schwabachanlage 6, Erlangen, Germany

7 Département d'Éthique de l'Université et des enseignements de Physiologie de la Faculté de Médecine PitiéSalpêtrière, 75013 Paris, France

8 Centre de Neuropathologie Est - Hospices Civils de Lyon, Université Claude Bernard Lyon1, Institut NeuroMyogène - CNRS UMR 5310 - INSERM U1217, Lyon, France 
9 Service d'Epileptologie Clinique, des Troubles du Sommeil et de Neurologie Fonctionnelle de l'Enfant, Hôpital Femme Mère Enfant, Lyon, France

10 Clinique neurologique et centre de référence des maladies rares neuromusculaires, Hôpital Roger-Salengro, CHRU de Lille, rue Emile-Laine, Lille, France

11 Centre de Référence des Maladies Neuromusculaires Nantes-Angers, Service de Neurologie, Centre Hospitalier Universitaire d'Angers, 49933 ANGERS

12 Centre Hospitalier Béthune Beuvry, Service des consultations externes pédiatrie, 62408 Béthune, France

13 Hospices Civils de Lyon, Hôpital Neurologique Pierre Wertheimer, Service d'ENMG-Pathologies neuromusculaires, 69677 Lyon-Bron, France

\section{*Corresponding author}

Present address: Stéphanie Bauché ICM UMRS1127, Hôpital de la Pitié-Salpêtrière, 47-83 boulevard de l’Hôpital, 75013 Paris, France ; Tanya Stojkovic AP-HP, Hôpital Pitié-Salpêtrière, 75013 Paris, France

* stephanie.godard-bauche@upmc.fr; Tel: +33-0157274411

*stojkovic.tanya@aphp.fr; Tel: +33-0142161932 


\begin{abstract}
Mutations in GFPT1 (glutamine-fructose-6-phosphate transaminase 1), a gene encoding an enzyme involved in glycosylation of ubiquitous proteins, cause a limb-girdle congenital myasthenic syndrome (LG-CMS) with tubular aggregates (TAs) characterized predominantly by affection of the proximal skeletal muscles and presence of highly organized and remodeled sarcoplasmic tubules in patients' muscle biopsies. We report here the first long-term clinical follow-up of 11 French individuals suffering from LG-CMS with TAs due to GFPT1 mutations, of which 9 are new. Our retrospective clinical evaluation stresses an evolution toward a myopathic weakness that occurs concomitantly to ineffectiveness of usual CMS treatments. Analysis of neuromuscular biopsies from 3 unrelated individuals demonstrates that the maintenance of neuromuscular junctions (NMJs) is dramatically impaired with loss of post-synaptic junctional folds and evidence of denervation-reinnervation processes affecting the 3 main NMJ components. Moreover, molecular analyses of the human muscle biopsies confirm glycosylation defects of proteins with reduced O-glycosylation and show reduced sialylation of transmembrane proteins in extrajunctional area. Altogether, these results pave the way for understanding the etiology of this rare neuromuscular disorder that may be considered as a "tubular aggregates myopathy with synaptopathy".
\end{abstract}

\title{
Keywords
}

Limb girdle myasthenia - GFPT1 - tubular aggregates - neuromuscular junction - myopathy 


\section{Introduction}

Congenital myasthenic syndromes (CMS) are a clinically and genetically heterogeneous group of inherited disorders that share common clinical features characterized by fluctuation of muscle weakness and fatigability resulting from defective synaptic transmission at the neuromuscular junction (NMJ) [1]. The distribution of symptoms and treatment's efficiency diverge according to the localization of the NMJ mutated proteins. So far, 31 genes have been described as causing the disease [2-9]. They encode proteins involved in pre-synaptic, synaptic or post-synaptic NMJ formation, maturation and maintenance. Among the clinical variability of CMS, the limb-girdle form (LG-CMS) is characterized by a muscle weakness and fatigability predominant in limbgirdle muscles with minimal or no involvement of ocular, bulbar and facial muscles [10]. Mutations in the DOK7 gene encoding the post-synaptic DOK7 kinase were first described to be responsible of this specific CMS subtype [11-14]. More recently, mutations in 5 additional genes were reported as causing LG-CMS. They encode for ubiquitous proteins involved in the glycosylation pathway such as GFPT1 (glutamine-fructose-6phosphate transaminase 1), DPAGT1 (dolichyl-phosphate N-acetylglucosaminephosphotransferase 1), ALG2 (alpha-1,3-mannosyltransferase), ALG14 (UDP-N-acetylglucosaminyltransferase subunit) and most recently GMPPB (GDP-mannose pyrophosphorylase B) [15-21]. Hallmarks of GFPT1-related LG-CMS are the presence of tubular aggregates (TAs) in patients' skeletal muscle biopsies and functional improvement with the use of acetylcholinesterase inhibitors (AChEI) to improve neuromuscular transmission [22, 23]. GFPT1 is the first and rate-limiting enzyme of the hexosamine biosynthetic pathway (HBP) involved in ubiquitous glycosylation processes [21]. HBP activation results in the production of UDP-N-acetylglucosamine (UDP-GlcNAc), a donor of $\mathrm{N}$-acetylglucosamine (GlcNAc) for O-linked and N-linked glycosylation to a wide range of proteins [24]. Interestingly, GFPT1 is expressed in various tissues, including skeletal muscle and motor nerve, raising the intriguing question of why mutations in such a ubiquitous gene would specifically lead to synaptic and muscular disorganization.

Since 2011, a total of 52 patients with GFPT1 mutations have been clinically reported [15, 21, 22, 25$]$. Yet, none of these studies was dedicated to a long-term clinical follow-up, and investigation of the endplate was only reported for 7 patients $[15,21,22,25]$. In this study, we report the retrospective clinical description and the molecular investigations of 11 individuals from 9 unrelated families with LG-CMS linked to recessivelyinherited mutations in GFPT1. We identified 9 new GFPT1 mutations, further stressing the high number of mutations reported in this gene associated with CMS and reinforcing that GFPT1 is the first genetic cause of 
ubiquitous CMS. We analyzed the synaptic features of muscle biopsies from 3 individuals confirming profound structural changes of endplates and highlighting an important instability of the NMJ with denervationreinnervation process. Moreover, we confirmed decreased glycosylation of muscle proteins and demonstrated that the cell surface muscle proteins sialylation is impaired in 4 human samples. These results suggest a global glycosylation deficiency that further emphasizes the reclassification of this form of CMS as "tubular aggregates myopathy with synaptopathy".

\section{Materials and methods}

Subjects and biological samples

Samples collection was performed in accordance with national and European regulations (Article L1243-3 and L1243-4). Participants gave informed consent through a prospective donor scheme approved by national ethic committees (DC-2012-1535 and AC-2012-1536). The 11 individuals from 9 families suffering from LG-CMS are Caucasian and offspring of non-consanguineous parents except for individual LGM2. All patients were followed up by electroneuromyography (ENMG) including repetitive nerve stimulation (RNS), nerve conduction studies and electromyography using standardized protocols [4]. Muscles of individuals without any neuromuscular disorder were used as controls.

Molecular analyses

Genomic DNA was isolated from blood samples by standard phenol-chloroform procedure or by using Qiasymphony DSP DNA Midi Kit on a Qiasymphony nucleic acid extraction machine (Qiagen). For five patients (LGM1, LGM2, LGM3, LGM4.1 and LGM6), the 19 exons of the GFPT1 gene were amplified by touchdown PCR on genomic DNA using the FastStart Taq polymerase (Roche Life Sciences) and sequenced by Sanger sequencing after enzymatic purification of PCR products (Promega) using standard procedures. The propositus of each family was used for initial screening, then the candidate variations were confirmed in the whole family when samples were available (primers, PCR and Sanger sequencing conditions are available on request). Sequences were analyzed using the DNA sequencing analysis (Applied Biosystems), Alamut ${ }^{\circledR}$ Visual (Interactive biosoftware) and MutationTaster (http://www.mutationtaster.org) softwares. Four patients (LGM5, LGM7.1, LGM8 and LGM9) were analyzed using targeted high-throughput sequencing. DNA was captured on an in-house SeqCap EZ library probe (Roche Nimblegen) containing exons and their intronic flanking regions of 
41 genes encoding critical proteins for skeletal muscle excitability and neuromuscular transmission, including GFPT1. The capture products were amplified and sequenced on a Miseq sequencer (Illumina®). The mean number of reads for targeted regions was higher than 500, thus allowing a reliable detection of copy number variations as well as point mutations. For two patients (LGM4.2 and LGM7.2), the mutations were directly searched after those identified in related patients (LGM4.1 and LGM7.1). For LGM1 patient, total RNAs were extracted from lymphoblastoid cell lines or skeletal muscle samples using Trizol reagent (Thermo Fisher Scientific). Reverse transcription was done using Thermoscript reverse transcriptase and random hexamers following the manufacturer's instructions (Thermo Fisher Scientific). PCR was done on reverse-transcribed cDNA using the FastStart Taq DNA polymerase (Roche Life Sciences) and was resolved using LabChipGX/GXII microfluidic system (Caliper LifeSciences). Sequencing of abnormal PCR products was done after agarose gel extraction procedures (NucleoSpin ${ }^{\circledR}$ gel and PCR clean-up, Macherey-Nagel). The NM_002056.3 Genbank sequence corresponding to the short isoform of GFPT1 was used as a reference.

Analyses of muscle biopsies

Muscle biopsies were taken from deltoid muscles by open biopsy. The NMJ-rich zone was determined by the small twitch provoked by the scalpel's tip on the muscle fascicles' surface. The presence of NMJs was confirmed on a longitudinal strip of the biopsy by revealing cholinesterase activity using the classical Koelle method [26]. Whole mounts of specimens fixed with $4 \%$ paraformaldehyde in PBS were stained for postsynaptic acetylcholine receptors (AChR) with TRITC-labeled $\alpha$-bungarotoxin ( $\alpha$-BGT, Molecular Probes), for terminal axon with monoclonal anti-neurofilament 165 kDa (2H3 clone, Hybridoma Bank) and 200 kDa (RT97 clone, Boeringer Ingelheim) antibodies and for terminal Schwann cells with a polyclonal anti-S100 antibody (Dako). The secondary antibodies were Alexa-488 conjugated anti-mouse or anti-rabbit IgG antibodies (ThermoFisher scientific). The specimens were mounted in Vectashield (Vector Laboratories) and observed using a LSM 510 Carl Zeiss confocal laser-scanning microscope equipped with a 63 objective (Zeiss, LSM 510).

Transversal cryosections of $10 \mu \mathrm{m}$ were stained with hematoxylin-eosin (H\&E), modified Gomori trichrome, NADH tetrazolium reductase (NADH-TR) and succinate dehydrogenase (SDH) and were analyzed for fiber morphology, fiber type distribution and accumulations. Immunofluorescent staining of lectin peanut agglutinin (PNA) and $\alpha$-dystroglycan was performed using Alexa-647 labelled PNA-lectin (Thermo Fisher scientific) and anti- $\alpha$-dystroglycan mouse antibody (clone IIH6, Santa Cruz Biotechnology), respectively. Slices were mounted 
in Vectashield (Vector Laboratories) and immunostaining was observed using a structured illumination fluorescence microscope (ApoTome 2) equipped with Zen2 software (Carl Zeiss).

For ultrastructural analysis, muscle samples were immediately fixed in a mixture of paraformaldehyde $2 \%$ and glutaraldehyde $2,5 \%$ in $0,12 \mathrm{M}$ phosphate buffer ( $\mathrm{pH} 7.4$ ). They were post-fixed in osmium tetroxide (2\%) for 2 hours in sodium cacodylate buffer, dehydrated in a graded series of ethanol and embedded in Epon resin. Ultrathin sections $(80 \mathrm{~nm}$ ) were contrasted with uranyl acetate followed by Reynolds lead citrate and viewed on a CM120 transmission electron microscope (Philips). Digital images were taken with a Morada CCD camera (Soft Imaging Systems).

Immunoblotting of GFPT1 and O-GlcNAc proteins

Transversal cryosections of muscles were collected in a sterile tube pre-cooled in dry ice. Tissues were resuspended in lysis buffer (1M Hepes, 10\% ASB-14, 2M NaCl, 1mg/mL Pepstatin and 1,74mg/mL PMSF), homogenized and centrifuged $10 \mathrm{~min}$ at $8000 \mathrm{rpm}$. Concentrations of solubilized proteins were determined using the BCA Protein Assay (Thermo Fisher Scientific). Samples containing equal amount of total proteins were prepared in Laemmli loading buffer (Thermo Fisher Scientific) and heated to $90^{\circ} \mathrm{C}$ during 5 min for SDS-PAGE electrophoresis. Samples were resolved in 7-5\% polyacrylamide gels (BioRad) and transferred to nitrocellulose membrane (GE Healthcare) overnight at $4^{\circ} \mathrm{C}$ for immunoblotting. Blots were blocked in TBS-1X (Euromedex) with $0,2 \%$ Tween 20 and 5\% milk for 1 hour and incubated in TBS1X with $0,2 \%$ Tween 20 and 5\% milk containing primary antibodies, monoclonal anti-GFPT1 (ab125069 clone, Abcam) or mouse anti O-GlcNAc (OLinked N-Acetylglucosamine, RL2 antibody, Abcam), overnight at $4^{\circ} \mathrm{C}$. Blots were rinsed 3 times with TBS1X and developed by chemiluminescence with HRP-conjugated goat anti-mouse antibodies (Jackson ImmunoResearch). Blots were revealed with Odyssey CLx infrared imaging system LI-COR (Biosciences).

\section{Results}

Clinical presentation

Among the 11 individuals from 9 families with LG-CMS investigated here (LGM1 to LGM9), 2 (LGM1 and LGM2) have been already described [27, 28]. All except LGM2 were from non-consanguineous families. Clinical data are summarized in Table 1. First symptoms of muscle weakness and/or fatigability developed during the first decade for 8 patients, except for 3 who became symptomatic at the age of 15, 22 and 24 years 
(LGM6, LGM7.1 and LGM5, respectively). All individuals first presented with a typical phenotype of LG-CMS with predominant symmetric proximal muscle weakness of the four limbs including quadriceps, glutei and iliopsoas muscles. Distal muscle weakness of tibialis anterior, foot and wrist appeared after the proximal muscle weakness in all individuals. Two individuals had flat feet (LGM6 and LGM8) and 2 (LGM5 and LGM9) had discreet Achilles retractions. Facial, oculomotor and bulbar weakness was moderate or absent for all. Half patients had axial weakness (neck flexor and/or abdominals). Bilateral scapular winging was observed in LGM9. All patients had fluctuations of their motor symptoms from a few days to a few months. The symptoms were generally stable until the fourth decade. Proximal muscle weakness worsened in LGM1 and LGM2 individuals from 50 and 45 years old respectively, leading to the use of canes or wheel chair. Furthermore, variation of intrafamilial severity was striking in LGM4 family. The oldest individual (LGM4.1) had a disease onset at the age of 6 and was still fully ambulatory at the age of 18, whereas her younger sister (LGM4.2) displayed worsening of the symptoms with a waddling gait and muscle weakness that necessitated the use of wheel chair since 8 years old. Psychomotor delay was noticed in one individual (LGM8), who was born with hip luxation and camptodactyly.

Repetitive nerve stimulation (RNS, $3 \mathrm{~Hz}$ ) resulted for all patients in a decrement of compound muscular action potential (CMAP) in proximal muscles such as trapezius. When recording from distal muscles such as tibialis anterior, RNS yielded a decrement in 6 patients (LGM1, LGM3, LGM4.1, LGM4.2, LGM6 and LGM9, Table 1). Single fiber EMG (SFEMG) was not done. Needle electromyography showed a myopathic pattern in proximal muscles (deltoids, biceps brachialis and/or quadriceps) for all individuals except for 3 of them (LGM6, LGM7.1 and LGM8). Motor and sensory nerve conduction was normal for all.

The initial diagnosis for all these patients was congenital or metabolic myopathy due to proximal muscle weakness and onset in childhood. Fatigability and decrement of CMAP in response to RNS then oriented toward CMS. Serum levels of creatine kinase (CK) were normal or slightly elevated ( 2 or $3 \mathrm{xN})$. Muscle biopsies were performed for diagnosis purpose for 6 patients and TAs were observed in all biopsies (Table 1). In LGM4.1 muscle biopsy, sarcoplasmic reticulum defects were observed using ultrastructural analyses and not routine histological investigations (data not shown).

All patients initially benefited from AChE inhibitors (AChEI) associated for some to 3,4-diaminopyridine (3,4DAP). However, it must be noticed that the benefit of these drugs gradually depleted within years and in the meanwhile the proximal motor weakness worsened. 
Sanger sequencing of GFPT1 identified a total of 12 mutations, among which 9 have never been described (Table 1 and Figure 1). All mutations were inherited and segregated with the disease. LGM2 and LGM7 patients were homozygous for one missense mutation, but only one is known to have related parents (LGM2). All the others harbored biallelic mutations.

Six of the 9 novel mutations encoded missense substitutions that were all predicted as disease causing. One variation (c.332G>A; p.Arg111His) was observed in 4 unrelated LG-CMS individuals of Caucasian origin (LGM1, LGM5, LGM6 and LGM8) and was reported in 3 heterozygous allele carriers in 1000G and ExAC database (rs189717232). This nucleotidic variation replaces the Arg111 amino acid residue, which is frequently observed as substituted by a Cys residue in the reported cohorts, by a His residue. This confirmed the hot spot status for mutation of this codon, which is located in the glutaminase aminotransferase type- 2 domain of GFPT1. The c.1496T > G mutation (p.Met499Arg) was reported in one allele carrier (rs147646112 heterozygous state) in human genome variation databases.

Furthermore, two novel splicing mutations not reported in any variant database were identified and predicted as disease causing. The c.207G>A silent mutation (LGM1) was located in exon 3 of GFPT1 and was predicted to create a cryptic acceptor splicing site with a score equal to 74.6 (Human Splicing Finder). We performed RTPCR on control and LGM1's lymphoblast mRNAs using primers encompassing exons 2 and 6 and confirmed abnormal splicing events of GFPT1 (data not shown). In control, a product of $400 \mathrm{bp}$ corresponding to the normal amplification of the exons 2-6 region was obtained. In LGM1 sample, additional bands of $1100 \mathrm{bp}, 900$ bp and a major $300 \mathrm{bp}$ products were observed. Sanger sequencing of this smaller PCR product revealed the complete deletion of exon 3 (data not shown). mRNAs lacking exon 3 are predicted to result in an in-frame deletion of 37 amino acids in the glutaminase aminotransferase type-2 domain (p.Gly39_Lys75delinsGlu). The heterozygous c.2002-1G>C transversion is located in the canonical acceptor-splicing site of the last intron of GFPT1. According to its predicted splicing impact, the molecular analysis of GFPT1 mRNA extracted from LGM4.1 muscle biopsy using one primer located within the 3' region of intron 18 showed the retention of this intronic region in GFPT1 mRNAs (data not shown). The exact impact of this intronic insertion could not be determined at the protein level.

The use of high-throughput sequencing for GFPT1 screening in LGM9 led to the identification of one heterozygous genomic deletion encompassing the targeted region of exon 11 (hg19 chr2:g.[6957298569573181]) with a 2-fold reduction of this region coverage compared to other samples (data not shown). The expected consequence is exon 11 deletion in mRNA, which is predicted to delete 32 amino acids of the linkage 
region located between the glutamine transferase domain and the first sugar isomerase domain (p.Gly319_Thr350del, see Figure 1A).

Neuromuscular junction abnormalities in GFPT1-related LG-CMS

To investigate the direct or indirect pathogenicity effect of GFPT1 mutations at the synaptic level, we performed whole-mount analyses of NMJs for 3 patients (LGM1, LGM3 and LGM4.1). They displayed fatigability or muscle weakness from 6 years old (LGM4.1) to 40 years old (LGM1). We found that pre- and post-synaptic architectures were disturbed in all deltoid muscle samples analyzed (25 NMJs/patient). Among the post-synaptic modifications, AChR fluorescent staining with $\alpha$-BTX appeared drastically decreased compared to control and showed structural modifications with fragmented and undefined gutters compared to the well-defined structure observed in the control sample (Figure 2A, a-d, i-1).

In regard to the previously poorly investigated pre-synaptic element, immunostaining of motor nerve with neurofilaments $(165 \mathrm{kDa}$ or $200 \mathrm{kDa})$ showed more than $50 \%$ of partially innervated NMJs that pointed on functional denervation-reinnervation processes contrary to control in which axonal branches typically innervated the well-defined postsynaptic structure (Figure 2A, e-h; Figure 2B). This was particularly prominent in LGM1 sample with abnormal terminal axons contacting small scattered synaptic cups (Figure 2A, b-f). A fully denervated profile was observed in LGM3 sample with lack of axonal staining and larger undefined postsynaptic gutters (Figure 2A, c-g). Moreover, the lack of well-defined synaptic gutters was associated with hypertrophy and cellular extensions of terminal Schwann cells (Figure 2A, m-p). The repartition of synaptic profiles into 4 categories (normal, denervated, remodelled and neoformed) confirmed that a great majority of NMJs were denervated or remodelled in the patients samples compared to the control samples (Figure 2B).

At the ultrastructural level, NMJs analyses (15 NMJs) performed in LGM1, LGM3 and LGM4.2 samples revealed a profound remodelling of endplates with absence (Figure 3A, arrows in LGM1 and LGM3) or loss of junctional folds (Figure 3A, LGM1 and LGM4.2). Representative pictures from LGM1 and LGM3 showed nerve terminals partially enwrapped by terminal Schwann cells (Figure 3A, stars in LGM1 and LGM3). TAs beneath the sarcolemma were observed in LGM1 and LGM3 samples (Figure 3B).

Decrease of GFPT1 and O-GlcNac levels is associated with reduced sialylation of transmembrane proteins in skeletal muscles 
GFPT1 is a key enzyme for synthesis of UDP-GlcNAc which serves as the substrate for N- and O-linked glycosylation. To evaluate whether the levels of GFPT1 were modified in muscle biopsies of LG-CMS individuals, we realized immunoblotting and observed a significant decrease of GFPT1 protein levels in the 4 samples analyzed (LGM1, 2, 3 and 4.1) compared to controls (Figure 4A). To confirm that the O-linked pathway was disturbed when GFPT1 is reduced, we performed western-blotting and observed a decrease of O-GlcNAc protein levels (cytosolic and nuclear proteins) for all patients' samples tested compared to controls (Figure 4B). Interestingly, a significant increase of sarcolemma staining of peanut agglutinin (PNA), a well-known tool for detecting decreased sialic acid level of membrane-associated glycoproteins, was observed on transversal sections of muscle biopsies from all tested LG-CMS samples compared to control (Figure 4C). This indicates either reduced sialylation or glycosylation of transmembrane proteins in the extra-junctional area. A defective glycosylation of $\alpha$-dystroglycan was recently reported in muscle samples of individuals with limb-girdle muscular dystrophy due to GDP-mannose pyrophosphorylase B (GMPPB) mutations [19, 20]. To investigate whether the glycosylation of $\alpha$-dystroglycan was also disrupted in individuals suffering from LG-CMS due to GFPT1 mutations, we performed immunostaining of glycosylated $\alpha$-dystroglycan in patients' muscle biopsies samples using the IIH6C antibody. We did not observe any significant change of immunostaining compared to control, suggesting distinct glycosylation changes between LG-CMS forms related to GFPT1 and GMPPB (Figure 4C).

\section{Discussion}

Fifty-two individuals with LG-CMS due to 41 distinct GFPT1 mutations have been reported in the literature since $2011[15,21,22,25]$. In this study, we reinforce the high number of loss-of-function GFPT1 mutations as causing LG-CMS with identification of 9 new mutations in 11 individuals from the French cohort of CMS. The clinical phenotype of all LG-CMS patients was characterized by a limb-girdle distribution of the muscle weakness, a decrement at $3 \mathrm{~Hz}$ RNS, TAs on muscle biopsy and an initial good response to treatment of fatigable muscle weakness with AChEI that decreased with years [10, 29]. For most individuals, diagnosis of myasthenia gravis was first evocated owing to fatigability and decrement at RNS. Diagnosis of congenital or metabolic myopathy was also considered due to the onset of proximal muscle weakness in childhood. The identification of TAs at muscle biopsy eventually led to a diagnosis of LG-CMS, further confirmed by the identification of GFPT1 mutations. Interestingly, we noticed thanks to our retrospective long-term follow-up, that the clinical course of the myopathic aspect of muscle weakness was concomitant to the decreased efficacy of 
AChEI and 3,4-DAP treatments for a large majority of individuals, strengthening the hypothesis that GFPT1related LG-CMS could be a congenital myopathy with synaptopathy.

Clinically distinguishing LG-CMS with TAs due to mutations in GFPT1 from those due to DPAGT1 is extremely difficult $[16,17,29]$. Furthermore, regarding LG-CMS due to mutations in $A L G 2$ and $A L G 14$, clinical signs are very similar to LG-CMS due to GFPT1 or DPAGT1 mutations with however a delay motor milestones and very few cases with TAs on their muscles biopsies for $A L G 2$ patients [18]. An extrajunctional hallmark of GFPT1 is the occurrence of TAs. These structures are organized with a large accumulation of tubules originating from the whole sarcoplasmic reticulum $[27,30]$. TAs are rarely observed in CMS except in individuals suffering from LG-CMS with mutations in GFPT1 [15, 21], DPAGT1 [16, 17] and ALG2 [18] and most rarely in individuals suffering from slow channel CMS [31]. The role of such structures in skeletal muscles as well as their pathological consequences are still subjects of debate $[32,33]$. However, the presence of TAs in muscle biopsies as histological hallmark associated with a clear-cut defect of neuromuscular transmission largely orients the clinical diagnosis toward GFPT1 or DPAGT1-related CMS [20, 21]. Furthermore, all patients with GFPT1 mutations containing TAs in ultrastructural and immunohistochemical analyses exhibited significant alterations of NMJs architecture. TAs are frequently associated with congenital neuromuscular diseases resulting in abnormal calcium handling [34] such as tubular aggregates myopathy (TAM) due to mutations in STIMI and ORAI1 [35-38] or periodic paralyses due to CACNA1S mutations [39]. This suggests that defective GFPT1 activity results in abnormal calcium homeostasis and TAs occurrence in an unknown way.

The reduced levels of GFPT1 in LG-CMS individuals have been proposed to decrease cell-surface accumulation of post-synaptic AChRs at the NMJ resulting in defective neuromuscular transmission [22, 40]. Yet, the structural impact exerted by decreased level of GFPT1 on the pre- and post-synaptic compartments in humans have been less well investigated $[15,22]$. Despite the high number of individuals with LG-CMS reported in the literature, the morphological investigation of NMJs — done by AChR fluorescent staining and ultrastructural analyses — was reported only for 7, among which one was considered as normal [15, 22]. In this study, we confirmed that reduced levels of GFPT1 induce a profound remodeling of endplates characterized by a loss of junctional folds and demonstrated for the first time that abnormal nerve endings are involved in an important denervation-reinnervation process with nerve terminals capped by hypertrophic terminal Schwann cells for 3 individuals. Furthermore, the number of NMJ analyzed (25 NMJs/patients), allows us to categorize the pre- and post-synaptic changes. These structural changes of NMJs were accompanied with functional modifications of the neurotransmission efficiency underlined by the CMAP decrement in response to $3 \mathrm{~Hz}$ RNS 
for all patients. As previously mentioned, GFPT1 is a key enzyme involved in the glycosylation pathway of proteins expressed in a wide range of cells including motoneurons and muscles fibers [21, 41]. Since postsynaptic AChRs are multimeric glycoproteins with all subunits being $\mathrm{N}$-linked glycosylated, defects in the muscle glycosylation pathway could explain NMJ morphological alterations observed in LG-CMS patients [40]. However, a glycomic study using primary muscle cells of 3 LG-CMS patients with GFPT1 mutations have recently shown that the global N-linked glycosylation was not significantly altered compared to controls, further supporting the hypothesis that the molecular mechanisms implicated in this rare neuromuscular disease do not exclusively rely on a muscular glycosylation deficit but rather is the result of global synaptic glycosylation defects [42].

Our molecular investigation confirmed that decreased levels of GFPT1 result in abnormal muscle protein glycosylation and showed for the first time altered sialylation of transmembrane muscle proteins in samples of individuals with LG-CMS. GFPT1 is essential for the synthesis of UDP-GlcNAc, through the hexosamine biosynthetic pathway (HBP), which serves as the substrate for glycosylation as well as for conversion into other nucleotide sugars such as fructose-6-phosphate to glucosamine-6-phosphate [24]. A defective glycosylation of $\alpha$-dystroglycan has been reported in individuals with GMPPB mutations or in dystroglycanopathy (MDDG), which was not observed in our study, stressing the distinct molecular impact of GMPPB and GFPT1 deficiencies [19, 20, 43]. Furthermore, individuals with GFTP1 and GMPPB mutations are undistinguishable at the clinical and electrophysiological levels, since both exhibit proximal muscle weakness and altered neurotransmission. They both respond to AChEI and 3-4 DAP and the benefit of these treatments turns to dull over time in both diseases while the motor weakness worsens gradually over years. This clinical observation strengthens the myopathic component in the LG-CMS form due to GFPT1 mutations. Furthermore, an increased level of PNA lectin staining around the sarcolemma was increased in all investigated muscle samples of LG-CMS individuals, which indicates a reduced sialylation of mucin core O-glycans. Such a reduced sialylation is observed in individuals with myopathy due to mutations in the gene encoding the UDP-GlcNAc 2epimerase $(G N E)$, with distal myopathy with rimmed vacuoles or with hereditary inclusion body myositis (hIBMs) [44-46]. In this context, it is worth to point out that in addition to reduced neurotransmission due to NMJs molecular changes, important biological and clinical similarities with certain forms of myopathies occur in GFPT1-related LG-CMS patients. Therefore, we propose that these molecular changes may in part explain the clinical evolution toward a myopathic weakness that occurs concomitantly to ineffectiveness of CMS treatments. 
To summarize, our study reports 9 new mutations of GFPT1 in LG-CMS, confirming the status of this gene as the primary genetic cause of ubiquitous CMS [3]. We highlight a profound remodeling of NMJs characterized by a loss of junctional folds as well as abnormal nerve endings when GFPT1 is decreased and impaired $\mathrm{N}$ - and O-glycosylation processes not only at the synapse but also in the extrajunctional area. Together with the clinical evolution of LG-CMS linked to GFPT1 towards a myopathic weakness phenotype with progressive ineffectiveness of usual CMS treatments, these histological and molecular results lead us to consider individuals with GFPT1 mutations as suffering from a "tubular aggregates myopathy with synaptopathy" rather than from the usual ubiquitous CMS due to reduced post-synaptic density of AChRs linked to hypoglycosylation.

\section{Acknowledgements}

We gratefully acknowledge the families for their invaluable contribution in this work. This study was supported by AFM-Téléthon (grant ID 20030) and “Investissements d'avenir',ANR-10-IAIHU-06 programs (IHU-AICM). We also thank Prof. Hanns Lochmuller and Yasmin Issop (Institute of Genetic Medicine, Newcastle University, UK) for fruitful discussions. We thank the Plateforme d'Imagerie Cellulaire Pitié Salpêtrière (PICPS) and the Service Commun de Microscopie Université PARIS DESCARTES for confocal microscopy as well as the DNA and Cell Banks of Généthon and CRBREFGENSEP, and the Celis, Histomics and Bioinformatics/Biostatistics (iCONICS) core facilities of the ICM for precious technical support. We also thank Hybridoma Bank for their anti-neurofilament antibody (2H3).

\section{Conflict of interest}

On behalf of all authors, the corresponding author states that there is no conflict of interest.

\section{References}

1. Engel AG, Shen X-M, Selcen D, Sine SM (2015) Congenital myasthenic syndromes: pathogenesis, diagnosis, and treatment. Lancet Neurol 14:420-434. doi: 10.1016/S1474-4422(14)70201-7

2. Tintignac LA, Brenner H-R, Rüegg MA (2015) Mechanisms Regulating Neuromuscular Junction Development and Function and Causes of Muscle Wasting. Physiol Rev 95:809-852. doi: 10.1152/physrev.00033.2014 
3. Beeson D (2016) Congenital myasthenic syndromes: recent advances. Curr Opin Neurol 29:565-571. doi: 10.1097/WCO.0000000000000370

4. Bauché S, O’Regan S, Azuma Y, et al (2016) Impaired Presynaptic High-Affinity Choline Transporter Causes a Congenital Myasthenic Syndrome with Episodic Apnea. Am J Hum Genet 99:753-761. doi: 10.1016/j.ajhg.2016.06.033

5. O’Grady GL, Verschuuren C, Yuen M, et al (2016) Variants in SLC18A3, vesicular acetylcholine transporter, cause congenital myasthenic syndrome. Neurology 87:1442-1448.

6. O’Connor E, Töpf A, Müller JS, et al (2016) Identification of mutations in the MYO9A gene in patients with congenital myasthenic syndrome. Brain 139:2143-2153. doi: 10.1093/brain/aww130

7. Shen X-M, Scola RH, Lorenzoni PJ, et al (2017) Novel synaptobrevin-1 mutation causes fatal congenital myasthenic syndrome. Ann Clin Transl Neurol 4:130-138. doi: 10.1002/acn3.387

8. Lam C-W, Wong K-S, Leung H-W, Law C-Y (2017) Limb girdle myasthenia with digenic RAPSN and a novel disease gene AK9 mutations. Eur J Hum Genet EJHG 25:192-199. doi: 10.1038/ejhg.2016.162

9. Salpietro V, Lin W, Delle Vedove A, et al (2017) Homozygous mutations in VAMPl cause a presynaptic congenital myasthenic syndrome. Ann Neurol. doi: 10.1002/ana.24905

10. Evangelista T, Hanna M, Lochmüller H (2015) Congenital Myasthenic Syndromes with Predominant Limb Girdle Weakness. J Neuromuscul Dis 2:S21-S29. doi: 10.3233/JND-150098

11. Müller JS, Herczegfalvi A, Vilchez JJ, et al (2007) Phenotypical spectrum of DOK7 mutations in congenital myasthenic syndromes. Brain J Neurol 130:1497-1506. doi: 10.1093/brain/awm068

12. Beeson D, Higuchi O, Palace J, et al (2006) Dok-7 mutations underlie a neuromuscular junction synaptopathy. Science 313:1975-1978. doi: 10.1126/science.1130837

13. Ben Ammar A, Petit F, Alexandri N, et al (2010) Phenotype genotype analysis in 15 patients presenting a congenital myasthenic syndrome due to mutations in DOK7. J Neurol 257:754-766. doi: 10.1007/s00415009-5405-y

14. Palace J (2012) DOK7 congenital myasthenic syndrome: DOK7 congenital myasthenic syndrome. Ann N Y Acad Sci 1275:49-53. doi: 10.1111/j.1749-6632.2012.06779.x

15. Guergueltcheva V, Müller JS, Dusl M, et al (2012) Congenital myasthenic syndrome with tubular aggregates caused by GFPT1 mutations. J Neurol 259:838-850. doi: 10.1007/s00415-011-6262-z

16. Belaya K, Finlayson S, Cossins J, et al (2012) Identification of DPAGT1 as a new gene in which mutations cause a congenital myasthenic syndrome. Ann N Y Acad Sci 1275:29-35. doi: 10.1111/j.17496632.2012.06790.x

17. Belaya K, Finlayson S, Slater CR, et al (2012) Mutations in DPAGT1 Cause a Limb-Girdle Congenital Myasthenic Syndrome with Tubular Aggregates. Am J Hum Genet 91:193-201. doi:

10.1016/j.ajhg.2012.05.022

18. Cossins J, Belaya K, Hicks D, et al (2013) Congenital myasthenic syndromes due to mutations in $A L G 2$ and ALG14. Brain 136:944-956. doi: 10.1093/brain/awt010

19. Belaya K, Rodríguez Cruz PM, Liu WW, et al (2015) Mutations in GMPPB cause congenital myasthenic syndrome and bridge myasthenic disorders with dystroglycanopathies. Brain 138:2493-2504. doi: 10.1093/brain/awv185

20. Montagnese F, Klupp E, Karampinos DC, et al (2017) Two patients with G MPPB mutation: The overlapping phenotypes of limb-girdle myasthenic syndrome and limb-girdle muscular dystrophy dystroglycanopathy: GMPPB Dystroglycanopathy. Muscle Nerve. doi: 10.1002/mus.25485 
21. Senderek J, Müller JS, Dusl M, et al (2011) Hexosamine Biosynthetic Pathway Mutations Cause Neuromuscular Transmission Defect. Am J Hum Genet 88:162-172. doi: 10.1016/j.ajhg.2011.01.008

22. Selcen D, Shen X-M, Milone M, et al (2013) GFPT1-myasthenia Clinical, structural, and electrophysiologic heterogeneity. Neurology 81:370-378.

23. Dusl M, Senderek J, Muller JS, et al (2015) A 3'-UTR mutation creates a microRNA target site in the GFPT1 gene of patients with congenital myasthenic syndrome. Hum Mol Genet 24:3418-3426. doi: $10.1093 / \mathrm{hmg} / \mathrm{ddv} 090$

24. Willems AP, van Engelen BGM, Lefeber DJ (2016) Genetic defects in the hexosamine and sialic acid biosynthesis pathway. Biochim Biophys Acta BBA - Gen Subj 1860:1640-1654. doi: 10.1016/j.bbagen.2015.12.017

25. Huh S-Y, Kim H-S, Jang H-J, et al (2012) Limb-girdle myasthenia with tubular aggregates associated with novel GFPT1 mutations. Muscle Nerve 46:600-604. doi: 10.1002/mus.23451

26. Couteaux R, Taxi J (1952) [Distribution of the cholinesterase activity at the level of the myoneural synapse]. Comptes Rendus Hebd Seances Acad Sci 235:434-436.

27. Chevessier F, Bauché-Godard S, Leroy J-P, et al (2005) The origin of tubular aggregates in human myopathies. J Pathol 207:313-323. doi: 10.1002/path.1832

28. Zephir H, Stojkovic T, Maurage CA, et al (2001) [Tubular aggregate congenital myopathy associated with neuromuscular block]. Rev Neurol (Paris) 157:1293-1296.

29. Finlayson S, Palace J, Belaya K, et al (2013) Clinical features of congenital myasthenic syndrome due to mutations in DPAGT1. J Neurol Neurosurg Psychiatry 84:1119-1125. doi: 10.1136/jnnp-2012-304716

30. Chevessier F, Marty I, Paturneau-Jouas M, et al (2004) Tubular aggregates are from whole sarcoplasmic reticulum origin: alterations in calcium binding protein expression in mouse skeletal muscle during aging. Neuromuscul Disord 14:208-216. doi: 10.1016/j.nmd.2003.11.007

31. Chaouch A, Müller JS, Guergueltcheva V, et al (2012) A retrospective clinical study of the treatment of slow-channel congenital myasthenic syndrome. J Neurol 259:474-481. doi: 10.1007/s00415-011-6204-9

32. Brady S, Healy EG, Gang Q, et al (2016) Tubular Aggregates and Cylindrical Spirals Have Distinct Immunohistochemical Signatures. J Neuropathol Exp Neurol 75:1171-1178. doi: 10.1093/jnen/nlw096

33. Schiaffino S (2012) Tubular aggregates in skeletal muscle: Just a special type of protein aggregates? Neuromuscul Disord 22:199-207. doi: 10.1016/j.nmd.2011.10.005

34. Takizawa S, Ishihara T, Shinohara Y (1986) [A case of hypokalemic periodic paralysis with tubular aggregates in type 2A fibers and type 2B fibers]. Rinsho Shinkeigaku 26:81-86.

35. Böhm J, Bulla M, Urquhart JE, et al (2017) ORAI1 Mutations with Distinct Channel Gating Defects in Tubular Aggregate Myopathy: HUMAN MUTATION. Hum Mutat 38:426-438. doi: 10.1002/humu. 23172

36. Böhm J, Chevessier F, De Paula AM, et al (2013) Constitutive Activation of the Calcium Sensor STIM1 Causes Tubular-Aggregate Myopathy. Am J Hum Genet 92:271-278. doi: 10.1016/j.ajhg.2012.12.007

37. Noury J-B, Böhm J, Peche GA, et al (2017) Tubular aggregate myopathy with features of Stormorken disease due to a new STIM1 mutation. Neuromuscul Disord NMD 27:78-82. doi: 10.1016/j.nmd.2016.10.006

38. Nesin V, Wiley G, Kousi M, et al (2014) Activating mutations in STIM1 and ORAI1 cause overlapping syndromes of tubular myopathy and congenital miosis. Proc Natl Acad Sci U S A 111:4197-4202. doi: 10.1073/pnas.1312520111 
39. Schartner V, Romero NB, Donkervoort S, et al (2017) Dihydropyridine receptor (DHPR, CACNA1S) congenital myopathy. Acta Neuropathol (Berl) 133:517-533. doi: 10.1007/s00401-016-1656-8

40. Zoltowska K, Webster R, Finlayson S, et al (2013) Mutations in GFPT1 that underlie limb-girdle congenital myasthenic syndrome result in reduced cell-surface expression of muscle AChR. Hum Mol Genet 22:2905-2913. doi: 10.1093/hmg/ddt145

41. Oki T, Yamazaki K, Kuromitsu J, et al (1999) cDNA cloning and mapping of a novel subtype of glutamine:fructose-6-phosphate amidotransferase (GFAT2) in human and mouse. Genomics 57:227-234. doi: 10.1006/geno.1999.5785

42. Chen Q, Müller J, Pang P-C, et al (2015) Global N-linked Glycosylation is Not Significantly Impaired in Myoblasts in Congenital Myasthenic Syndromes Caused by Defective Glutamine-Fructose-6-Phosphate Transaminase 1 (GFPT1). Biomolecules 5:2758-2781. doi: 10.3390/biom5042758

43. Carss KJ, Stevens E, Foley AR, et al (2013) Mutations in GDP-Mannose Pyrophosphorylase B Cause Congenital and Limb-Girdle Muscular Dystrophies Associated with Hypoglycosylation of $\alpha-$ Dystroglycan. Am J Hum Genet 93:29-41. doi: 10.1016/j.ajhg.2013.05.009

44. Tajima Y, Uyama E, Go S, et al (2005) Distal myopathy with rimmed vacuoles: impaired O-glycan formation in muscular glycoproteins. Am J Pathol 166:1121-1130. doi: 10.1016/S0002-9440(10)62332-2

45. Voermans NC, Guillard M, Doedee R, et al (2010) Clinical features, lectin staining, and a novel GNE frameshift mutation in HIBM. Clin Neuropathol 29:71.

46. Cerino M, Gorokhova S, Béhin A, et al (2015) Novel Pathogenic Variants in a French Cohort Widen the Mutational Spectrum of GNE Myopathy. J Neuromuscul Dis 2:131-136. doi: 10.3233/JND-150074 


\section{Legends to figures}

Figure 1 Schematic representation of the short isoform of GFPT1 and localization of all mutations identified to date in individuals suffering from LG-CMS.

(A) Mutations identified in the 11 patients reported here are underlined and the novel ones are in red. The muscle-specific exon incorporating 17 additional amino acids in the GATase-2 domain is in green. The amino acids encoded by exon 11 that would be deleted in individual LGM9 are in red. Nucleotidic and amino acid numbers are based on the short isoform of GFPT1 (NM-002056.3 and its translation product). GATase-2 = glutamine amidotransferase type 2 domain; SIS = sugar isomerase domains.

Figure 2 Morphological study of neuromuscular junctions contained in the muscle biopsy samples of 3 unrelated individuals with GFPT1 mutations.

(A) Representative whole-mount pictures of muscle biopsies stained in green both for motor axons with an antineurofilament (NF $165 \mathrm{kDa}$ ) antibody (e-h) and for the terminal Schwann cells with an anti-S100 antibody (m-p) and in red for postsynaptic AChRs with $\alpha$-bungarotoxin (a-d, i-l). In NMJs from the control sample, the axonal branchs typically end as a fork innervating a well-defined AChR structure (a, e, i, m). In LGM1 sample, abnormal neurofilament accumulation was seen in nerve terminal contacting small AChRs clusters, suggesting formation of novel NMJs (neoformation in $\mathrm{b}, \mathrm{f}, \mathrm{j}, \mathrm{n}$ ). Samples from LGM3 and LGM4.1 individuals showed an example of denervated NMJs (LGM3; c, g, k, o) or NMJs in denervation process (LGM4.1; d, h, 1, p) with large undefined synaptic gutters, thin terminal axons contacting the post-synaptic element and hypertrophic terminal Schwann cells $(\mathrm{k}, \mathrm{o}, \mathrm{i}, \mathrm{p})$. Scale bar $=10 \mu \mathrm{m}$ (applies to all pictures). (B) Histogram of NMJs classification into 4 groups. Changes in NMJs distribution with a majority of remodeled and denervated NMJs were observed in all LGM samples tested compared to the control (percentage of total number of NMJs analyzed, $\mathrm{N}=25$ for each sample).

Figure 3 Ultrastructural analyses of muscle biopsies of 3 unrelated individuals with LG-CMS due to GFPT1 mutations.

(A) Focus on the junctional area (15 NMJs/patient) showed a major remodelling of endplates with absence (arrows for LGM1 and LGM3) or loss of junctional folds (for LGM1 and LGM4.2) and partial enwrapping of nerve terminals by terminal Schwann cells (stars for LGM1 and LGM3). In some NMJs, well-formed post- 
synaptic folds were observed but they contained myeloid bodies (LGM1). (B) Ultrastructural analyses of extrajunctional area showed TAs beneath the sarcolemma (LGM1 and LGM3). Scale bar are indicated in all pictures.

Figure 4 Glycosylation impairment is associated with decrease of GFPT1 in muscle samples of individuals with LG-CMS due to GFPT1 mutations.

(A) Western-blot analysis of GFPT1 in muscle samples of LG1, LG2, LG3 and LG4.1 compared to 3 controls $(\mathrm{C} 1, \mathrm{C} 2, \mathrm{C} 3)$. A decreased amount of GFPT1 at the expected size was observed in all patients' samples tested compared to the controls. Coomassie blue staining was used as control of equal loading. (B) Western-blot analysis of O-GlcNac. A decreased amount of O-GlcNac proteins was noticed in all LG-CMS samples (LG1, LG2, LG3 and LG4.1) compared to the control. (C) Immunohistochemistry of glycosylated $\alpha$-dystroglycan (in green) and fluorescent staining of PNA lectin (in red) in muscle biopsies of 4 LG-CMS patients and one control. A normal staining was observed for the glycosylated forms of $\alpha$-dystroglycan recognized by the IIH6 antibody in all LG-CMS samples, whereas an increased staining with PNA lectin was observed in all LG-CMS samples tested (LG1, LG2, LG3 and LG4.1) compared to one control. The muscle fibers of the LG1 patient are hypertrophic. Scale bar $=200 \mu \mathrm{m}$ (applied to all pictures). 
Figure 1

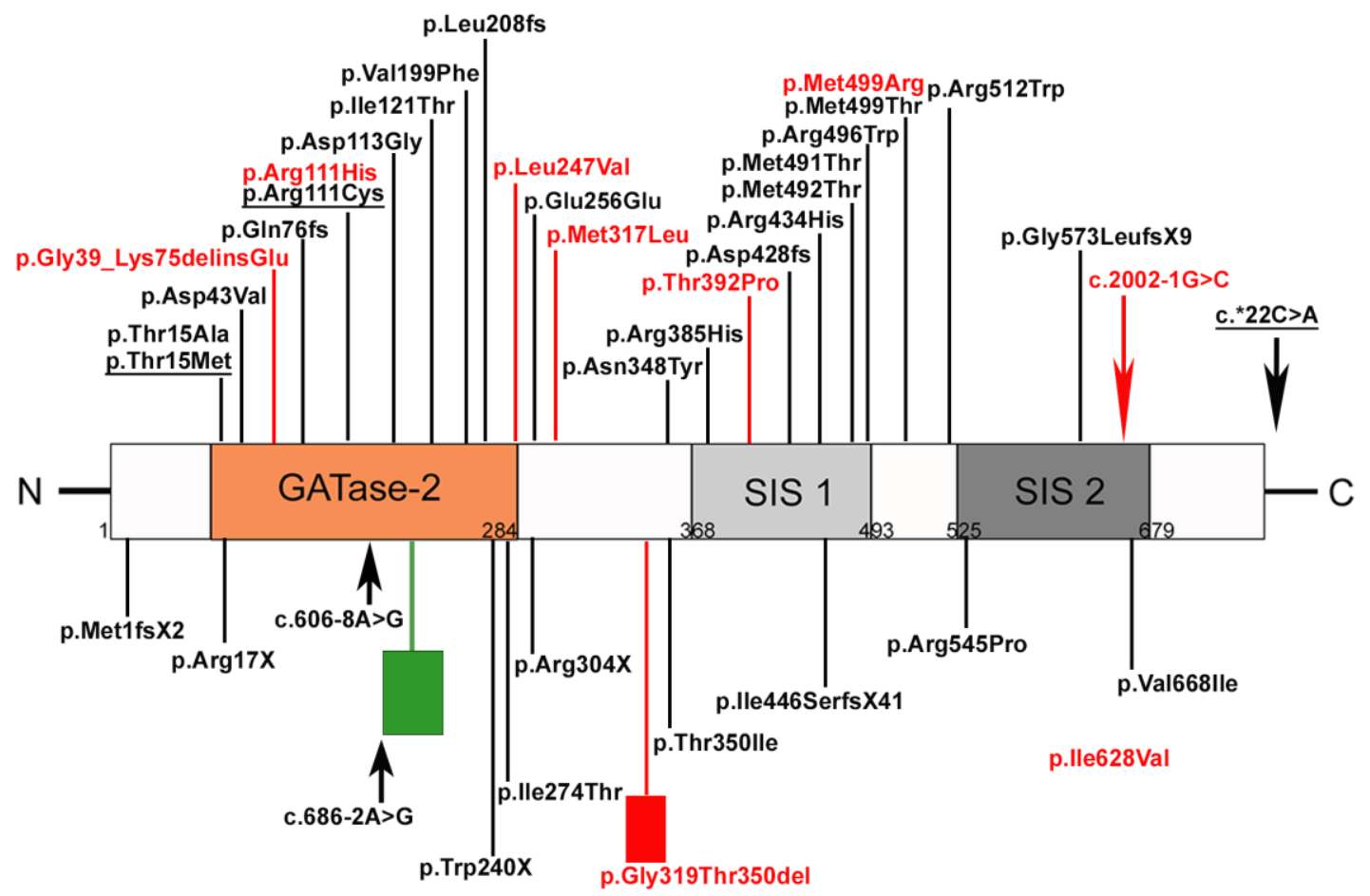


Figure 2

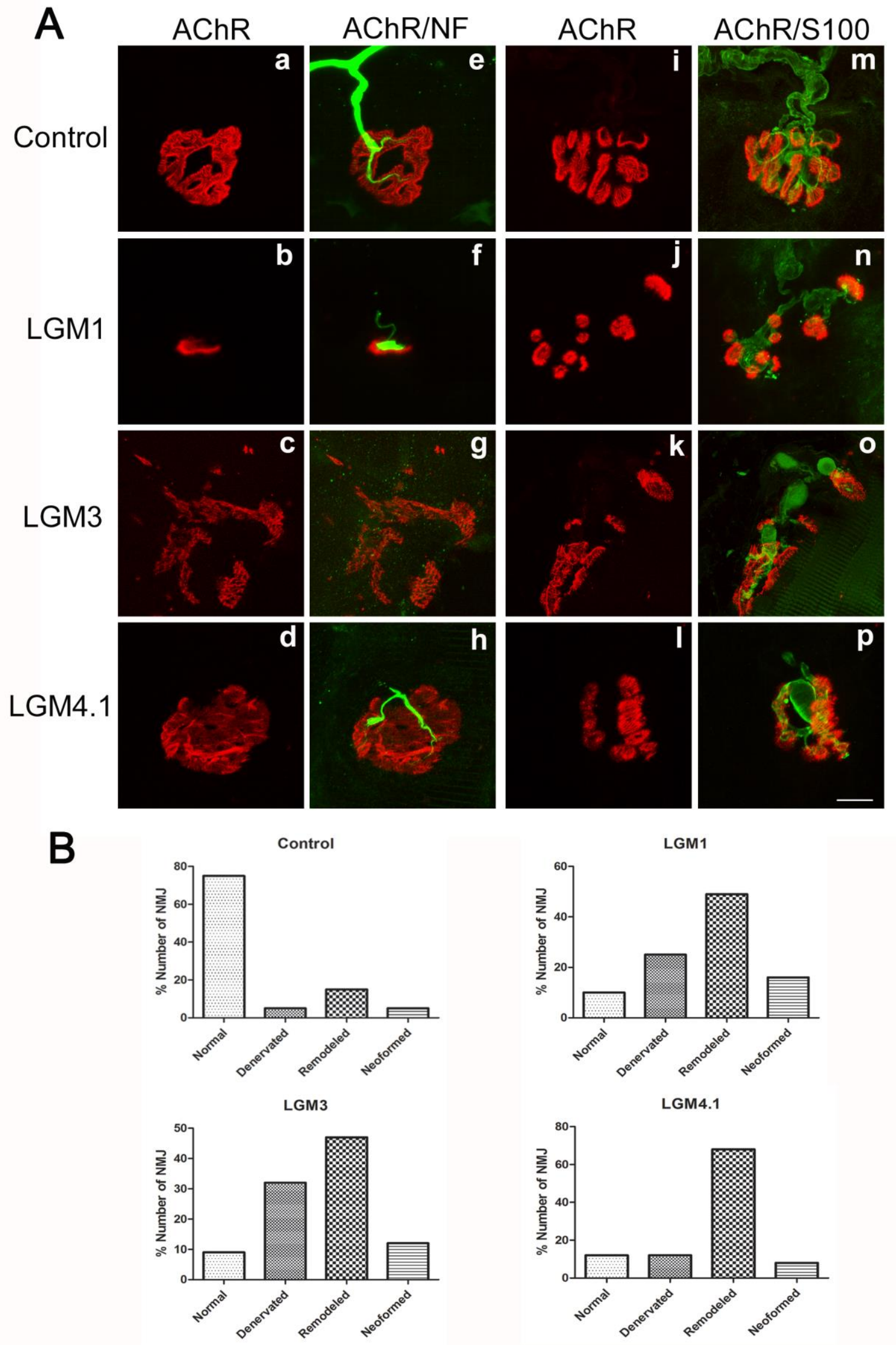


Figure 3

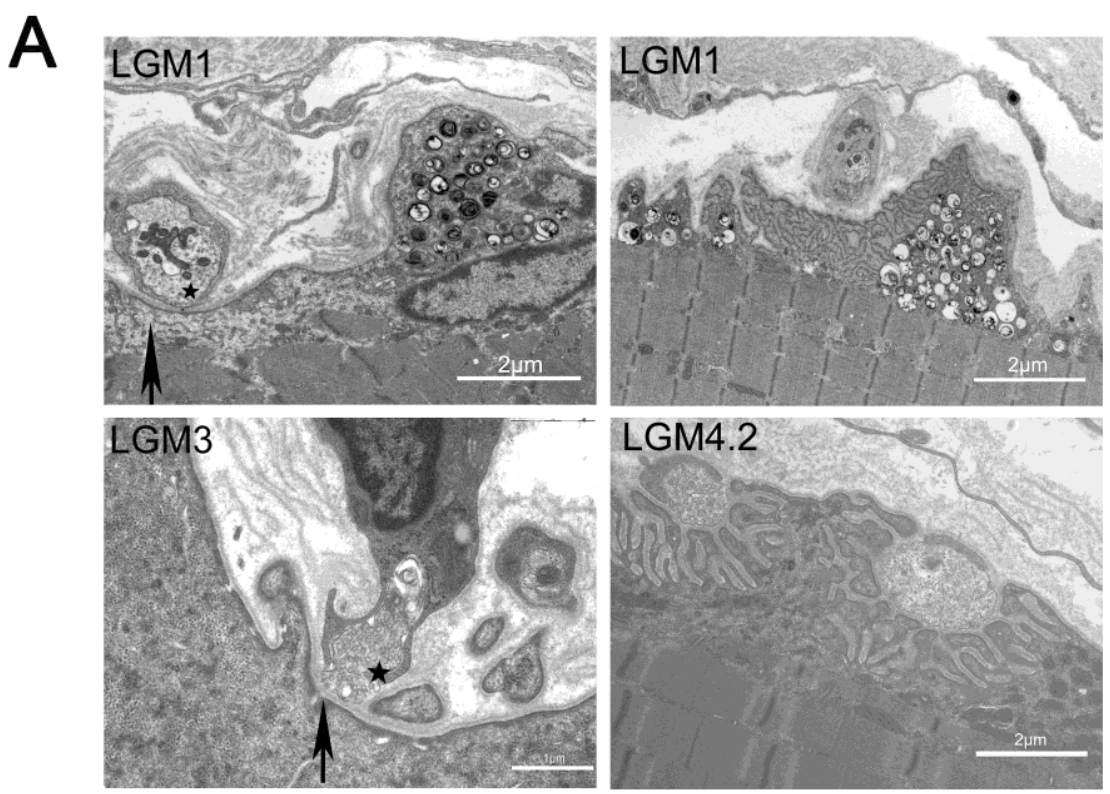

B
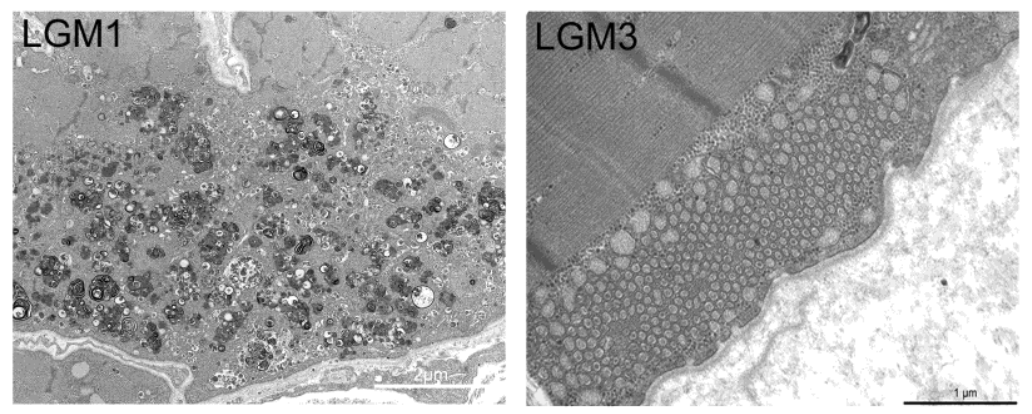
Figure 4
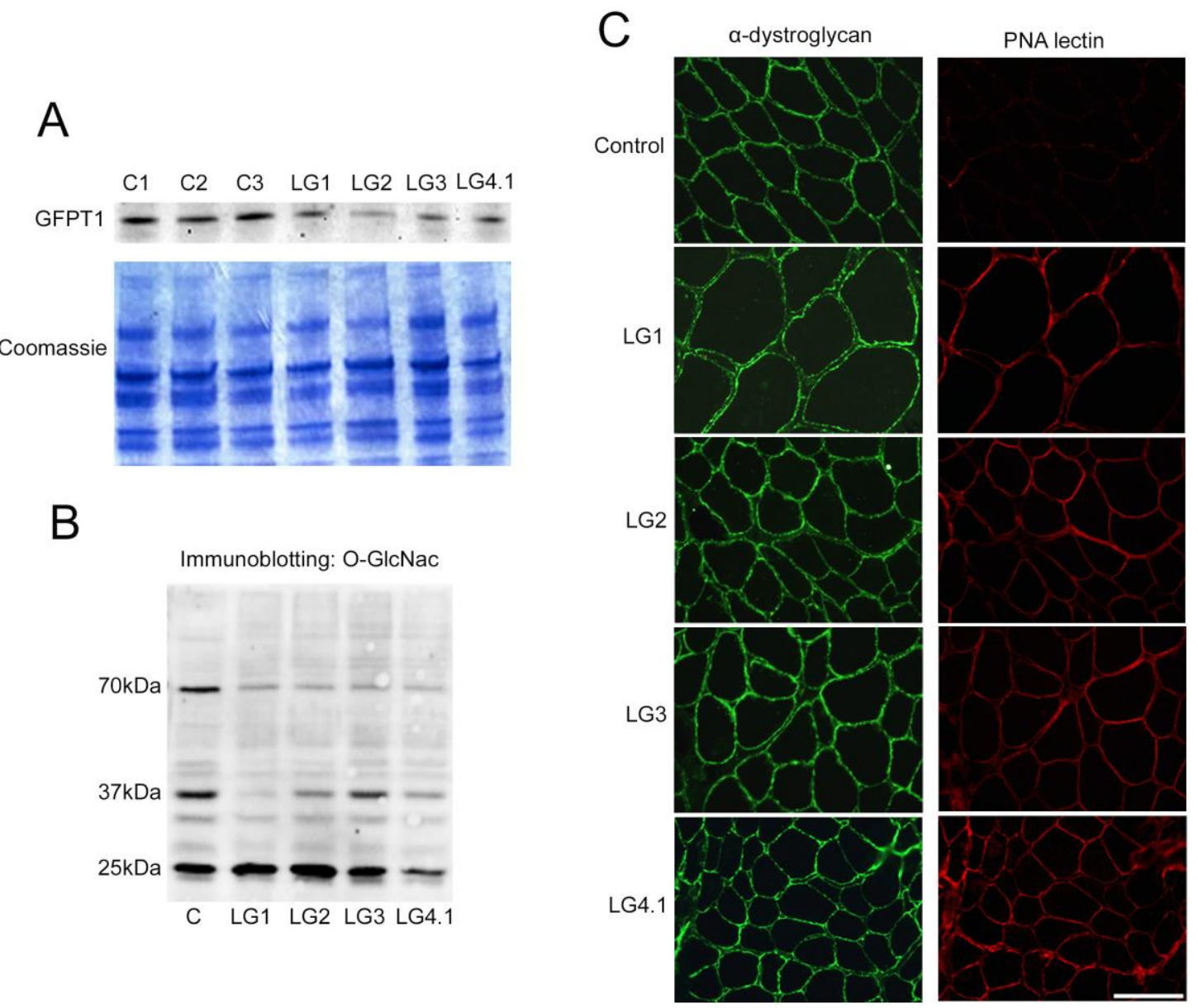
Table 1: Clinical features from LG-CMS patients described in this study

\begin{tabular}{|c|c|c|c|c|c|c|c|c|c|c|c|}
\hline Patient & LGM1 & LGM2 & LGM3 & LGM4.1 & LGM4.2 & LGM5 & LGM6 & LGM7.1 & LGM7.2 & LGM8 & LGM9 \\
\hline $\begin{array}{l}\text { Nucleotidic } \\
\text { mutation }\end{array}$ & $\begin{array}{l}\text { c. } 207 \mathrm{G}>\mathrm{A} \\
\text { c. } 332 \mathrm{G}>\mathrm{A}\end{array}$ & $\begin{array}{l}\text { c. } 331 C>T \\
\text { c. } 331 C>T\end{array}$ & $\begin{array}{l}\text { c. } 1174 A>C \\
\text { c. } 1496 T>G\end{array}$ & $\begin{array}{l}\text { c. } 331 \mathrm{C}>\mathrm{T} \\
\text { c. } 2002-1 \mathrm{G}>\mathrm{C}\end{array}$ & $\begin{array}{l}c .331 C>T \\
c .2002-1 G>C\end{array}$ & $\begin{array}{l}\text { c. } 332 \mathrm{G}>\mathrm{A} \\
\text { c. }{ }^{*} 22 \mathrm{C}>\mathrm{A}\end{array}$ & $\begin{array}{l}\text { c.332G }>A \\
\text { c.949A }>\mathrm{T}\end{array}$ & $\begin{array}{l}\text { c. } 44 C>T \\
\text { c. } 44 C>T\end{array}$ & $\begin{array}{l}\text { c. } 44 C>T \\
\text { c. } 44 C>T\end{array}$ & $\begin{array}{l}\text { c. } 332 \mathrm{G}>\mathrm{A} \\
\text { c. } 739 \mathrm{C}>\mathrm{G}\end{array}$ & $\begin{array}{l}\text { c. } 1882 A>G \\
\text { c. }(955+1956- \\
1)(1051+1105 \\
2-1) \text { del }\end{array}$ \\
\hline $\begin{array}{l}\text { Protein } \\
\text { variations }\end{array}$ & $\begin{array}{l}\text { p.Gly39_Lys75 } \\
\text { delinsGlu; } \\
\text { p.Arg111His }\end{array}$ & $\begin{array}{l}\text { p.Arg111Cys } \\
\text { p.Arg111Cys }\end{array}$ & $\begin{array}{l}\text { p.Thr392Pro; } \\
\text { p.Met499Arg }\end{array}$ & $\begin{array}{l}\text { p. Arg111Cys } \\
\text { to be } \\
\text { determined }\end{array}$ & $\begin{array}{l}\text { p. Arg111Cys } \\
\text { to be } \\
\text { determined }\end{array}$ & $\begin{array}{l}\text { p.Arg111His; } \\
\text { 3'UTR }\end{array}$ & $\begin{array}{l}\text { p.Arg111His; } \\
\text { p.Met317Leu }\end{array}$ & $\begin{array}{l}\text { p.Thr15Met } \\
\text { p.Thr15Met }\end{array}$ & $\begin{array}{l}\text { p.Thr15Met } \\
\text { p.Thr15Met }\end{array}$ & $\begin{array}{l}\text { p.Arg111His; } \\
\text { p.Leu247Val }\end{array}$ & $\begin{array}{l}\text { p.lle628Val; } \\
\text { p.Gly319Thr3 } \\
\text { 50del** }\end{array}$ \\
\hline Age at onset & First decade & 1 year & 6 years & 6 years & 2,5 years & 24 years & 15 years & 22 years & First decade & First decade & First decade \\
\hline Age at report & 68 years & 49 years & 18 years & 16 years & 15 years & 32 years & 21 years & 27 years & 29 years & 15 years & 58 years \\
\hline $\begin{array}{l}\text { Symptoms at } \\
\text { onset }\end{array}$ & $\begin{array}{c}\text { Muscle } \\
\text { weakness and } \\
\text { fatigability }\end{array}$ & $\begin{array}{c}\text { Muscle } \\
\text { weakness }\end{array}$ & Fatigability & Fatigability & $\begin{array}{c}\text { Muscle } \\
\text { weakness }\end{array}$ & $\begin{array}{c}\text { Muscle } \\
\text { weakness and } \\
\text { fatigability }\end{array}$ & $\begin{array}{c}\text { Muscle } \\
\text { weakness }\end{array}$ & $\begin{array}{c}\text { Muscle } \\
\text { weakness and } \\
\text { fatigability }\end{array}$ & $\begin{array}{c}\text { Muscle } \\
\text { weakness }\end{array}$ & Fatigability & $\begin{array}{c}\text { Muscle } \\
\text { weakness and } \\
\text { fatigability }\end{array}$ \\
\hline $\begin{array}{l}\text { Symptoms } \\
\text { course }\end{array}$ & $\begin{array}{l}\text { Worsening } \\
\text { since } 50 \text { years } \\
\text { (before and } \\
\text { after therapy) }\end{array}$ & $\begin{array}{c}\text { Worsening } \\
\text { since } 45 \text { years }\end{array}$ & $\begin{array}{c}\text { Muscle } \\
\text { weakness }\end{array}$ & Stable & $\begin{array}{c}\text { Worsening } \\
\text { then stability } \\
\text { in the last } 4 \\
\text { years }\end{array}$ & $\begin{array}{c}\text { Slight } \\
\text { worsening }\end{array}$ & $\begin{array}{c}\text { Slight } \\
\text { worsening, } \\
\text { fatigability }\end{array}$ & Variable & $\begin{array}{l}\text { Worsening } \\
\text { since } 12 \\
\text { years, } \\
\text { fatigability }\end{array}$ & Variable & $\begin{array}{c}\text { Worsening } \\
\text { since } 40 \text { years }\end{array}$ \\
\hline $\begin{array}{l}\text { Limb-girdle } \\
\text { weakness }\end{array}$ & Yes & Yes & Yes & Yes & Yes & Yes & Yes & Yes & Yes & Yes & Yes \\
\hline Fluctuation & Yes & Yes & Yes & Yes & Yes & Yes & Yes & Yes & Yes & Yes & Yes \\
\hline $\begin{array}{l}\text { Facial/bulbar } \\
\text { /ocular/other } \\
\text { symptom }\end{array}$ & No/no/no/no & No/Mild/No/No & $\begin{array}{c}\text { No/no/no/ } \\
\text { no }\end{array}$ & $\begin{array}{c}\text { No/no/slight } \\
\text { transient } \\
\text { ptosis/no }\end{array}$ & No/no/no/no & $\begin{array}{c}\mathrm{No} / \mathrm{no} / \\
\text { intermittent } \\
\text { right } \\
\text { ptosis/no }\end{array}$ & No/no/no/no & No/no/no/no & No/no/no/no & $\begin{array}{c}\text { No/no/ ptosis } \\
\text { strabismus/ } \\
\text { psychomotor } \\
\text { delay }\end{array}$ & $\begin{array}{l}\text { Mild/no/mild } \\
\text { ptosis/no }\end{array}$ \\
\hline $\begin{array}{l}\text { Severity at } \\
\text { last } \\
\text { examination }\end{array}$ & $\begin{array}{c}\text { Wheel chair } \\
\text { since } 63 \text { years }\end{array}$ & $\begin{array}{l}\text { Ambulatory, } \\
\text { walks with } \\
\text { canes }\end{array}$ & $\begin{array}{c}\text { Mild } \\
\text { ambulatory } \\
\text { without aid }\end{array}$ & $\begin{array}{c}\text { Mild } \\
\text { ambulatory } \\
\text { without aid }\end{array}$ & Wheel chair & $\begin{array}{l}\text { Ambulatory } \\
\text { without aid }\end{array}$ & $\begin{array}{l}\text { Ambulatory } \\
\text { without aid }\end{array}$ & $\begin{array}{l}\text { Ambulatory } \\
\text { without aid }\end{array}$ & $\begin{array}{l}\text { Ambulatory } \\
\text { without aid }\end{array}$ & $\begin{array}{l}\text { Ambulatory } \\
\text { without aid }\end{array}$ & $\begin{array}{c}\text { Mild } \\
\text { ambulatory } \\
\text { without aid }\end{array}$ \\
\hline CK levels & 1,5 to $3 \mathrm{~N}$ & $2 \mathrm{~N}$ & $1,5 \mathrm{~N}$ & $\mathrm{~N}$ & $\mathrm{~N}$ & $4 \mathrm{~N}$ & $2 \mathrm{~N}$ & $\mathrm{~N}$ & $\mathrm{~N}$ & $2 \mathrm{~N}$ & $2 \mathrm{~N}$ \\
\hline $\begin{array}{l}\text { EMG } \\
\text { decrement at } \\
\text { RNS ( } 3 \mathrm{~Hz}) \text {, } \\
\% / \text { muscle }\end{array}$ & $\begin{array}{c}\text { 20/trapezius } \\
\text { 44/quadriceps } \\
\text { 21/ADM } \\
\text { 38/TA }\end{array}$ & 50/trapezius & $\begin{array}{c}\text { 52/trapezius } \\
\text { 49/anconeus } \\
\text { 45/TA }\end{array}$ & $\begin{array}{c}\text { 34/trapezius } \\
\text { 38/anconeus } \\
\text { 33/TA }\end{array}$ & $\begin{array}{c}\text { 50/trapezius } \\
\text { 54/anconeus } \\
\text { 19/ADM } \\
\text { 57/TA }\end{array}$ & $\begin{array}{l}\text { 54/trapezius } \\
\text { 44/anconeus }\end{array}$ & $\begin{array}{c}\text { 68/trapezius } \\
\text { 28/anconeus } \\
\text { 40/TA }\end{array}$ & $\begin{array}{l}\text { 20/trapezius } \\
\text { 14/anconeus }\end{array}$ & 25/trapezius & $\begin{array}{l}\text { 34/trapezius } \\
\text { 20/anconeus }\end{array}$ & $\begin{array}{c}\text { 21/trapezius } \\
\text { 22/anconeus } \\
\text { 30/TA }\end{array}$ \\
\hline
\end{tabular}




\begin{tabular}{|c|c|c|c|c|c|c|c|c|c|c|c|}
\hline $\begin{array}{l}\text { Response to } \\
\text { AChEl at last } \\
\text { examination }\end{array}$ & ++ & ++ & ++ & ++ & ++ & + & ++ & ++ & ++ & ND & ND \\
\hline $\begin{array}{l}\text { Response to } \\
3.4-D A P \text { at } \\
\text { last } \\
\text { examination }\end{array}$ & $\begin{array}{c}++ \\
\text { Decreased } \\
\text { with time }\end{array}$ & $\begin{array}{c}++ \\
\text { Decreased } \\
\text { with time }\end{array}$ & $\begin{array}{c}++ \\
\text { Decreased } \\
\text { with time }\end{array}$ & ++ & + & ++ & $\begin{array}{c}++ \\
\text { Decreased with } \\
\text { time }\end{array}$ & ++ & ++ & ND & $\mathrm{ND}$ \\
\hline $\begin{array}{l}\text { Tubular } \\
\text { aggregates in } \\
\text { muscle biopsy }\end{array}$ & Yes & Yes & Yes & Yes & Yes & ND & Yes & ND & ND & ND & ND \\
\hline
\end{tabular}

ND: not done; N: Normal; AChEI: acetylcholinesterase inhibitors; 3,4-DAP: 3,4-diaminopyridine; ADM: Abductor Digiti Minimi; TA: Tibialis Anterior; +++ = full

improvement,$++=$ moderate improvement, $+=$ slight improvement; $* *$ predicted impact of exon 11 deletion 